\title{
Hydrogen Sulfide Improves Vascular Calcification in Rats by Inhibiting Endoplasmic Reticulum Stress
}

\author{
Rui Yang, ${ }^{1}$ Xu Teng, ${ }^{1,2}$ Hui Li, ${ }^{1}$ Hong-Mei Xue, ${ }^{1}$ Qi Guo, ${ }^{1}$ Lin Xiao, ${ }^{1}$ and Yu-Ming Wu ${ }^{1,3,4}$ \\ ${ }^{1}$ Department of Physiology, Institute of Basic Medicine, Hebei Medical University, Shijiazhuang 050017, China \\ ${ }^{2}$ Hebei Key Lab of Laboratory Animal Science, Hebei Medical University, Shijiazhuang 050017, China \\ ${ }^{3}$ Hebei Collaborative Innovation Center for Cardio-Cerebrovascular Disease, Shijiazhuang 050017, China \\ ${ }^{4}$ Key Laboratory of Vascular Medicine of Hebei Province, Shijiazhuang 050017, China
}

Correspondence should be addressed to Yu-Ming Wu; wuyum@yahoo.com

Received 27 November 2015; Revised 21 January 2016; Accepted 1 February 2016

Academic Editor: Kenneth R. Olson

Copyright (c) 2016 Rui Yang et al. This is an open access article distributed under the Creative Commons Attribution License, which permits unrestricted use, distribution, and reproduction in any medium, provided the original work is properly cited.

In this study, the vitamin $\mathrm{D}_{3}$ plus nicotine (VDN) model of rats was used to prove that $\mathrm{H}_{2} \mathrm{~S}$ alleviates vascular calcification (VC) and phenotype transformation of vascular smooth muscle cells (VSMC). Besides, $\mathrm{H}_{2} \mathrm{~S}$ can also inhibit endoplasmic reticulum stress (ERS) of calcified aortic tissues. The effect of $\mathrm{H}_{2} \mathrm{~S}$ on alleviating VC and phenotype transformation of VSMC can be blocked by TM, while PBA also alleviated VC and phenotype transformation of VSMC that was similar to the effect of $\mathrm{H}_{2} \mathrm{~S}$. These results suggest that $\mathrm{H}_{2} \mathrm{~S}$ may alleviate rat aorta VC by inhibiting ERS, providing new target and perspective for prevention and treatment of VC.

\section{Introduction}

Vascular calcification (VC) refers to the abnormal deposition of calcium and phosphorus minerals on the walls of blood vessels. The phenomenon of VC is common in atherosclerosis, hypertension, diabetic vascular disease, aging, and chronic kidney disease, and an important risk factor of cardiocerebrovascular diseases and related deaths [1]. There has been no clinical therapy specifically developed to treat VC.

The deposition of calcium mineral in VC was initially considered to occur passively. However, according to recent studies VC is actually an active biological process similar to bone development that is highly regulated, preventable, and can be reversed. In particular, the development and progression of VC involve the transformation of vascular smooth muscle cells (VSMCs) from a contractile to an osteoblast-like phenotype [2-5]. An agent that could prevent this transformation would have great guiding significance in research and potential for clinical application.

Recent studies have revealed that paracrine/autocrine vasoactive factors such as cardiovascular peptides [6], cytokines, and gaseous molecules [7] have an important regulatory role in the development and progression of VC. Hydrogen sulfide $\left(\mathrm{H}_{2} \mathrm{~S}\right)$ is newly identified as a gaseous signaling molecule after discovery of nitric oxide and carbon monoxide. $\mathrm{H}_{2} \mathrm{~S}$ affects the cardiovascular system in multiple ways, by dilating blood vessels, lowering blood pressure [8], and regulating the apoptosis and proliferation of VSMCs. Recent studies have suggested that $\mathrm{H}_{2} \mathrm{~S}$ also inhibits $\mathrm{VC}$ and the phenotype transformation of VSMCs [9]. However, the specific mechanism is unknown. The thorough investigation of the mechanism of the ameliorative effect of $\mathrm{H}_{2} \mathrm{~S}$ on VC should contribute to the transformation from bench to bedside and be useful to discover new target and strategy for treatment of VC.

The endoplasmic reticulum is the largest intracellular organelle of the cell. A disturbance of homeostasis in the endoplasmic reticulum leads to a series of cellular responses cumulatively known as endoplasmic reticulum stress (ERS). ERS has been implicated in the development and progression of a variety of cardiovascular diseases, and the development and progression of VC. The apoptosis of VSMCs that is caused by ERS promotes $\mathrm{VC}$, and also the phenotype transformation of VSMCs [10-12]. In addition, recent studies have suggested 
that $\mathrm{H}_{2} \mathrm{~S}$ may exert a protective effect on the cardiovascular system by inhibiting ERS [13-15]. Therefore, we hypothesized that $\mathrm{H}_{2} \mathrm{~S}$ may alleviate VC by inhibiting ERS.

\section{Materials and Methods}

2.1. Rats and Reagents. All animal procedures complied with the Animal Management Rule of the Ministry of Health, People's Republic of China (documentation number 55, 2001) and the Care and Use of Laboratory Animals published by the United States National Institutes of Health (NIH Publication number 85-23, revised 1996) and approved by the Animal Care Committee of Hebei Medical University.

Male Sprague-Dawley rats (180-200 g) were supplied by the Animal Center of Hebei Medical University (Shijiazhuang, China) and housed under standard conditions (room temperature $20 \pm 8^{\circ} \mathrm{C}$, humidity $60 \pm 10 \%$, lights from 600 to $1800 \mathrm{~h}$ ). They were freely supplied with standard rodent chow and water.

Tunicamycin (Tm) was from Cayman Chemical (Ann Arbor, USA). The alkaline phosphatase (ALP) and calcium detection kit was from BioSino Biotechnology and Science (Beijing, CN). Antibodies against calponin, RUNX2 (runt-related transcription factor 2), and GRP78 (78-kDa glucose-regulated protein) were from Epitomic (Burlingame, USA). Antibodies against SM22 $\alpha$, BMP2 (bone morphogenetic protein 2), active caspase-12, and beta-actin were from GeneTex (Irvine, USA). Antibodies against CHOP (C/EBP homologous protein) were from Affinity Biosciences (Cincinnati, USA). The secondary antibodies were from Kirkegaard \& Perry Laboratories (Gaithersburg, USA). An enhanced chemiluminescence (ECL) kit was from MultiSciences Biotech (Hangzhou, CN). Other chemicals and reagents were of analytical grade.

2.2. The Model of VC In Vivo. Male Sprague-Dawley rats $(180-200 \mathrm{~g})$ were randomly divided into 7 groups $(n=6$, each) as follows: the control; vitamin $\mathrm{D}_{3}$ plus nicotine (VDN); $\mathrm{VDN}+$ sodium hydrosulfide (NaHS); VDN + tunicamycin (Tm); VDN + Tm + NaHS; VDN + 4-phenylbutyric acid (PBA); and VDN + PBA + NaHS. In these groups, VDN treatment imposed calcification and therefore the VC model [16], $\mathrm{NaHS}$ acted as an $\mathrm{H}_{2} \mathrm{~S}$ donor, Tm acted as an ERS agonist, and PBA as an ERS inhibitor.

The rats in the noncontrol groups were given vitamin $\mathrm{D}_{3}$ (300000 IU/kg, intramuscularly) simultaneously with nicotine $(25 \mathrm{mg} / \mathrm{kg}$ in $5 \mathrm{~mL}$ peanut oil, intragastrically) at 08:00 hours on the first day. The nicotine administration was repeated at 2000 hours. On days 2 and 15, the rats were retreated with vitamin $\mathrm{D}_{3}$.

In the rat groups that received NaHS (i.e., VDN + NaHS, $\mathrm{VDN}+\mathrm{Tm}+\mathrm{NaHS}$, and VDN + PBA + NaHS), the NaHS was injected daily $(56 \mu \mathrm{mol} / \mathrm{kg})$ during the 28 days of nicotine treatment. In the rat groups that received Tm (VDN + Tm and $\mathrm{VDN}+\mathrm{Tm}+\mathrm{NaHS}$ ), Tm was injected daily (intraperitoneal, $5 \mu \mathrm{g} / \mathrm{kg}$ ) during the same period. In the rat groups that received $\mathrm{PBA}(\mathrm{VDN}+\mathrm{PBA}$ and $\mathrm{VDN}+\mathrm{PBA}+\mathrm{NaHS}), \mathrm{PBA}$ was injected daily (intraperitoneal, $50 \mathrm{mg}$ per rat) during the same period. Rats in the control group received normal saline intramuscularly (rather than vitamin $\mathrm{D}_{3}$ ) and 2 gavages of peanut oil ( $5 \mathrm{~mL} / \mathrm{kg}$, i.e., without nicotine) during the same period. The rats of the control and VDN groups were injected with saline vehicle $(0.2 \mathrm{~mL} / 100 \mathrm{~g})$ per day, at a volume similar to the volumes of NaHS, Tm, and/or PBA injected into the rats of the other groups, during the same period. The aortic tissues of all the rats were harvested on day 28 , as described below.

2.3. Blood Pressure Measurement. At the end of the 28day experimental period, the rats were anesthetized with urethane (intraperitoneal, $1 \mathrm{~g} / \mathrm{kg}$ ). A cannula was inserted into the right carotid artery to measure blood pressure. Systolic blood pressure was measured using a pressure transducer (BL420F-Powerlab, TaiMeng, Chengdu, China). After measurement, the blood and aortas were collected, and the aortas were stripped of intima and adventitia and treated as described below.

2.4. Hematoxylin and Eosin (HङE) Staining. To quantify vascular medial calcification, the aortic roots (from the aortic opening to $0.5 \mathrm{~cm}$ from the opening section of the aorta) were separated and stored in 4\% paraformaldehyde for $\mathrm{H} \& \mathrm{E}$ histopathological staining.

2.5. Quantification of Calcium Content and ALP Activity in Aortas. Calcium levels were determined by colorimetry through a reaction with o-cresolphthalein complexone. ALP activity was measured using an ALP Colorimetric assay kit, in accordance with the kit's instructions.

2.6. Western Blot Analysis. Aortas were homogenized in lysis buffer. Equal amounts of protein samples were loaded on $10 \%$ or $12 \%$ sodium dodecyl sulfate gels and then transferred to a nitrocellulose membrane. Nonspecific proteins were blocked with $5 \%$ nonfat dried milk for $1 \mathrm{~h}$. Membranes were incubated serially with the primary antibodies overnight at $4^{\circ} \mathrm{C}$, and with secondary antibody (horseradish peroxidaseconjugated anti-goat or anti-rabbit IgG) for $1 \mathrm{~h}$. The reaction was visualized by enhanced chemiluminescence, and an autoradiograph was scanned. Protein concentrations were analyzed using NIH Image software and normalized to that of $\beta$-actin. All experiments were repeated at least 3 times.

2.7. Measurement of Plasma $\mathrm{H}_{2} \mathrm{~S}$ Levels. Whole blood sample was collected, anticoagulated, and centrifuged at $3000 \mathrm{rpm}$ for $15 \mathrm{~min}$ to obtain the plasma. $\mathrm{H}_{2} \mathrm{~S}$ levels in plasma were measured as Shen et al. reported [17]. $\mathrm{H}_{2} \mathrm{~S}$ levels were calculated using a standard curve generated from a sodium sulfide solution $(0-100 \mu \mathrm{M})$.

2.8. Statistical Analyses. Statistical analyses were performed using GraphPad software (GraphPad Prism v5.00 for Windows; GraphPad Software, San Diego, CA, USA). Comparisons between 2 groups were conducted using the unpaired Student's $t$-test. Comparisons among $\geq 3$ groups were analyzed by one-way analysis of variance and then by NewmanKeuls test. Data are expressed as mean \pm standard deviation. $P<0.05$ was considered statistically significant. 
TABLE 1: General characteristics and vascular total calcium and plasma and aortic ALP.

\begin{tabular}{lcccc}
\hline & SBP & $\begin{array}{c}\text { Calcium } \\
\text { Aorta, mmol/g DW }\end{array}$ & $\begin{array}{c}\text { ALP } \\
\text { Plasma, IU/L }\end{array}$ & $\begin{array}{c}\text { ALP } \\
\text { Aorta, IU/g protein }\end{array}$ \\
\hline Control & $118.1 \pm 3.7$ & $6.11 \pm 2.21$ & $135.4 \pm 18.2$ & $250.20 \pm 60.22$ \\
VDN & $155.8 \pm 5.1^{\mathrm{a}}$ & $51.38 \pm 5.12^{\mathrm{a}}$ & $349.4 \pm 31.6^{\mathrm{a}}$ & $462.42 \pm 61.17^{\mathrm{a}}$ \\
$\mathrm{VDN}+\mathrm{NaHS}$ & $114.3 \pm 5.3^{\mathrm{b}}$ & $13.14 \pm 8.56^{\mathrm{b}}$ & $174.4 \pm 23.6^{\mathrm{b}}$ & $193.52 \pm 23.34^{\mathrm{b}}$ \\
VDN + Tm & $151.7 \pm 2.6^{\mathrm{a}, \mathrm{c}}$ & $60.56 \pm 10.01^{\mathrm{a}, \mathrm{c}}$ & $361.2 \pm 26.8^{\mathrm{a}, \mathrm{c}}$ & $510.46 \pm 41.10^{\mathrm{a}, \mathrm{c}}$ \\
VDN + Tm + NaHS & $150.0 \pm 4.9^{\mathrm{a}, \mathrm{c}}$ & $55.56 \pm 6.34^{\mathrm{a}, \mathrm{c}}$ & $340.6 \pm 18.0^{\mathrm{a}, \mathrm{c}}$ & $490.20 \pm 26.48^{\mathrm{a}, \mathrm{c}}$ \\
VDN + PBA & $110.3 \pm 3.3^{\mathrm{b}}$ & $14.01 \pm 5.78^{\mathrm{b}}$ & $125.4 \pm 31.2^{\mathrm{b}}$ & $221.56 \pm 22.46^{\mathrm{b}}$ \\
VDN + PBA + NaHS & $108 \pm 9.9^{\mathrm{b}}$ & $13.89 \pm 8.02^{\mathrm{b}}$ & $133.0 \pm 28.0^{\mathrm{b}}$ & $216.08 \pm 31.64^{\mathrm{b}}$ \\
\hline
\end{tabular}

${ }^{\mathrm{a}} P<0.05$ cf. control group; ${ }^{\mathrm{b}} P<0.05 \mathrm{cf}$. VDN; ${ }^{\mathrm{c}} P<0.05 \mathrm{cf}$. VDN $+\mathrm{H}_{2} \mathrm{~S}$ group.

SBP, systolic blood pressure.

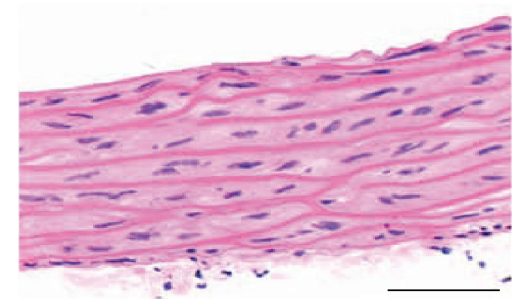

(a)

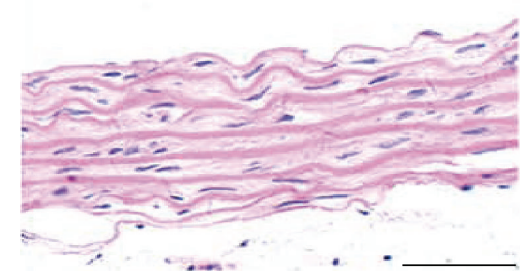

(d)

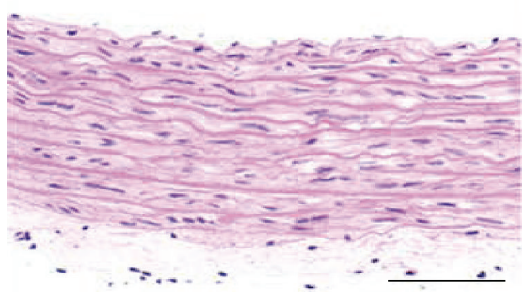

(b)

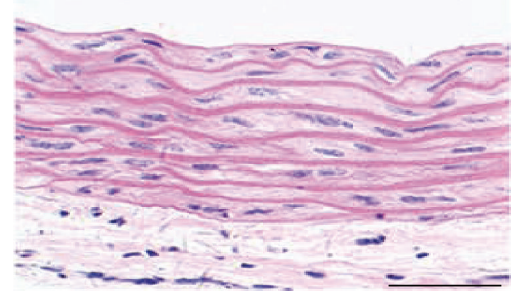

(e)

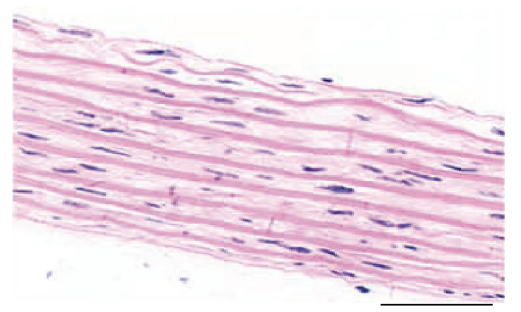

(g)

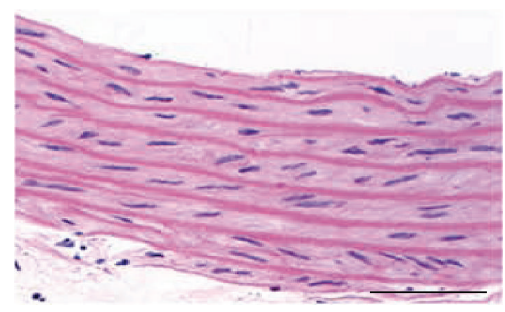

(c)

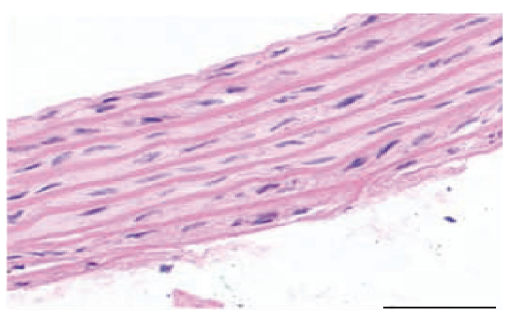

(f)

FIGURE 1: H\&E staining of the aorta in rats (400x). (a) Control group. (b) VDN group. (c) VDN + NaHS group. (d) VDN + Tm group. (e) $\mathrm{VDN}+\mathrm{Tm}+\mathrm{NaHS}$ group. (f) VDN + PBA group. (g) VDN + PBA + NaHS group. Scale bar is $50 \mu \mathrm{m}$.

\section{Results}

3.1. Comparison of VDN and Control Rats. In the VDN (nontreated VC model) rat group, the mean systolic blood pressure, ALP activity, and calcium content of aortal tissues were significantly higher relative to that of the control rats (Table 1). Furthermore, in the VDN group, H\&E staining revealed thickening and structural disorder of vascular elastic fibers (Figure 1), and the Western blot results showed that the protein levels of SM22 $\alpha$ and calponin, that is, molecular markers of a VSMC contractile phenotype, were significantly lower than that of the controls. Conversely, in the VDN group the protein levels of BMP2 and RUNX2, which indicate a VSMC osteoblast-like phenotype, were significantly higher than that of the controls (Figure 2).

Compared with the control rats, in the VDN rats the protein levels of the ERS markers GRP78, active caspase-12, and CHOP, were all significantly higher (Figure 3).

3.2. Comparison of VDN + NaHS and VDN Rats. In the $\mathrm{VDN}+\mathrm{NaHS}$ group, relative to the VDN rats the NaHS treatment was associated with higher systolic blood pressure and aortal tissue ALP activity, and calcium content was lower (Table 1). Furthermore, relative to the VDN rats, rats in 


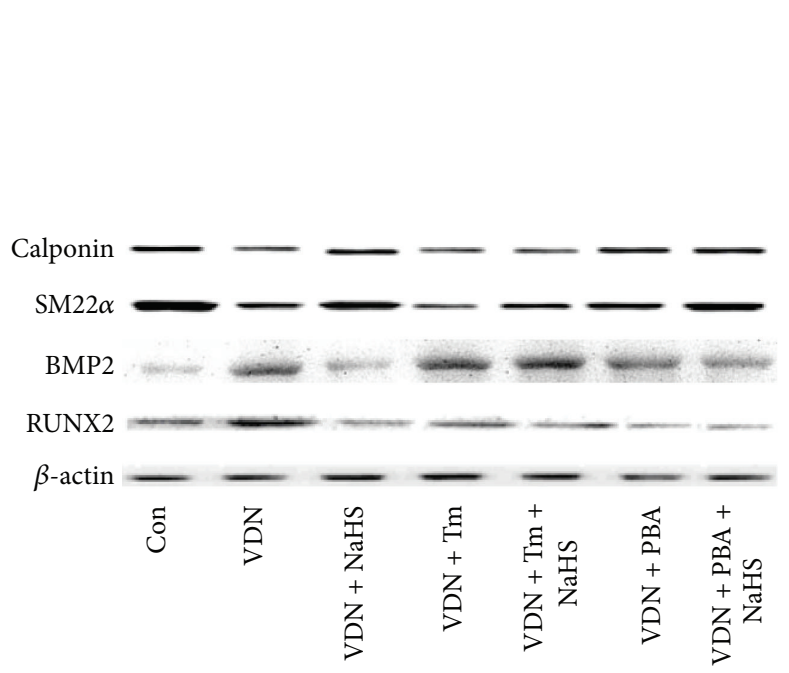

(a)

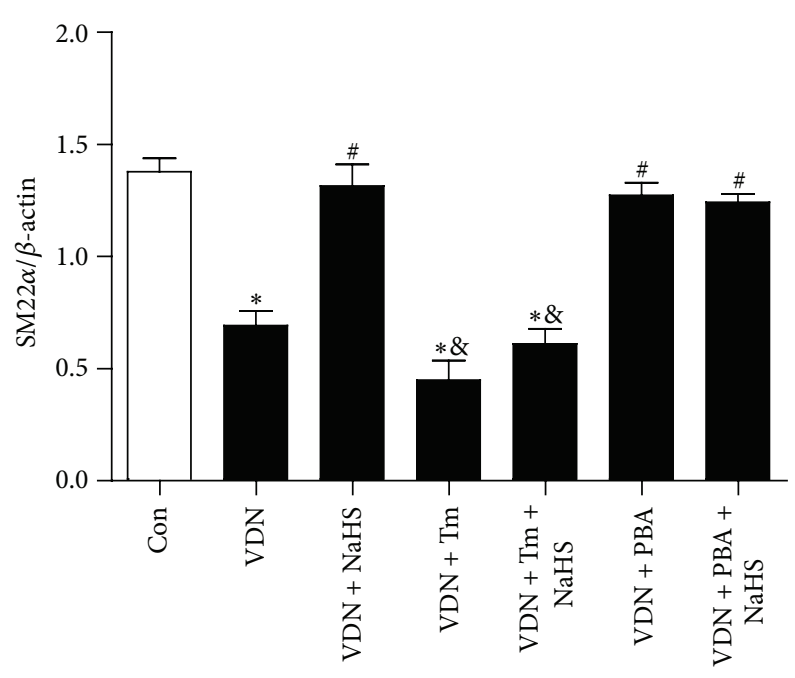

(c)

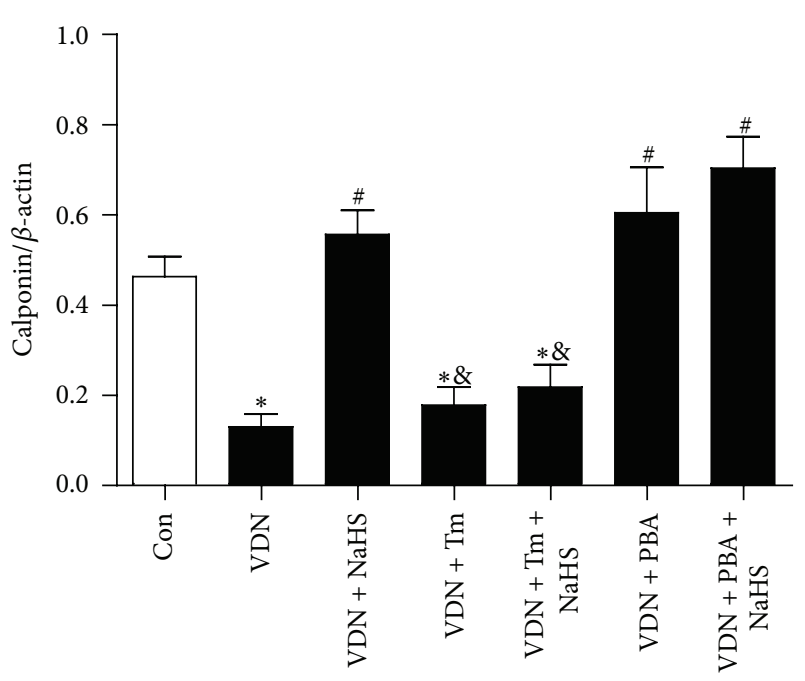

(b)

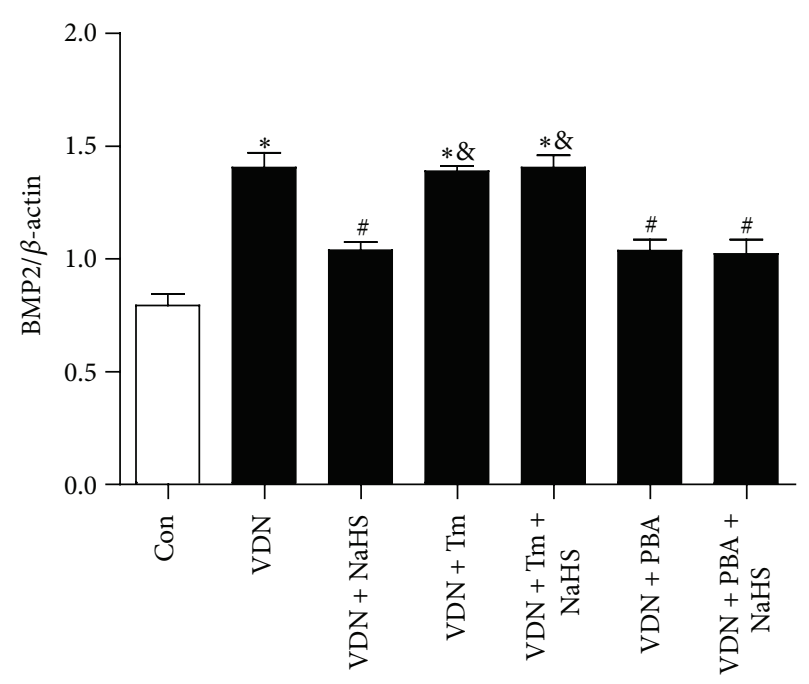

(d)

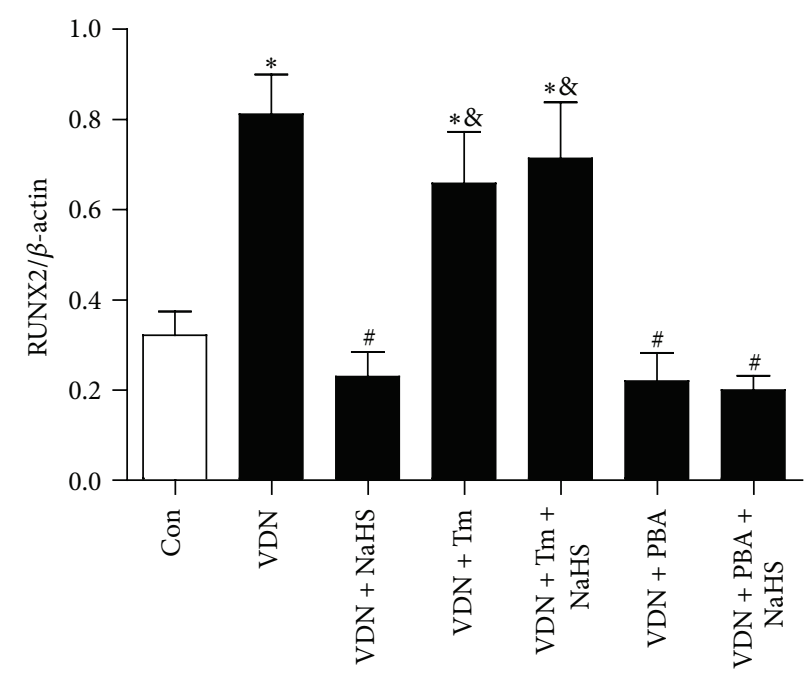

(e)

FIGURE 2: Tm blocks the effect of $\mathrm{H}_{2} \mathrm{~S}$ on transformation in VSMCs in calcified aorta. (a) Representative protein levels of calponin, SM22 $\alpha$, BMP2, RUNX2, and $\beta$-actin; (b-e) quantitative analysis of protein levels of calponin, SM22 $\alpha, \mathrm{BMP} 2$, and RUNX2. ${ }^{*} P<0.05 \mathrm{cf}$. control group; ${ }^{\#} P<0.05 \mathrm{cf}$. VDN group; ${ }^{\&} P<0.05 \mathrm{cf}$. VDN + NaHS group. 


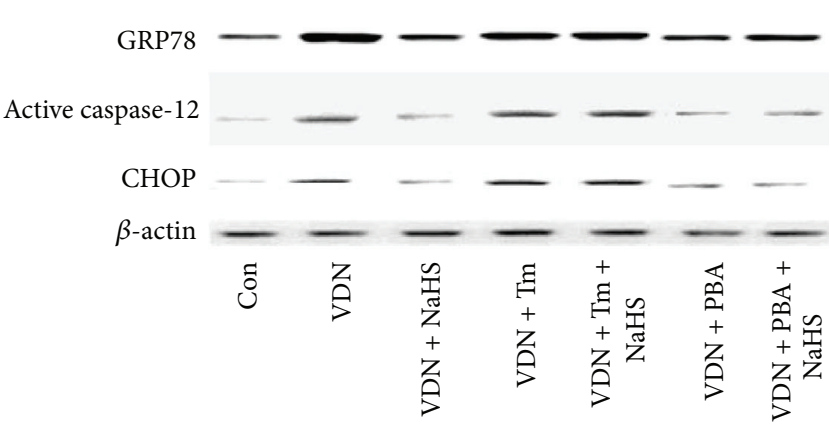

(a)

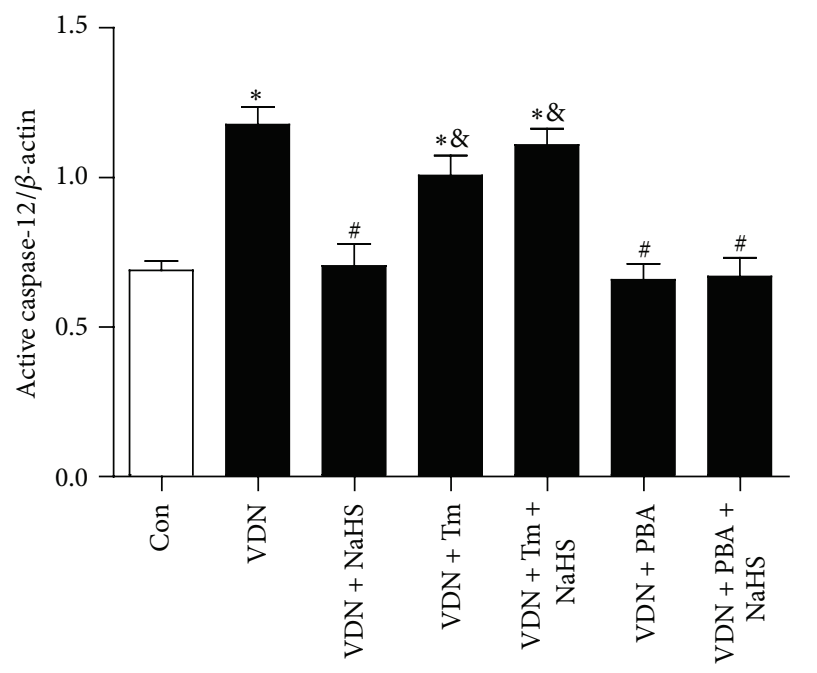

(c)

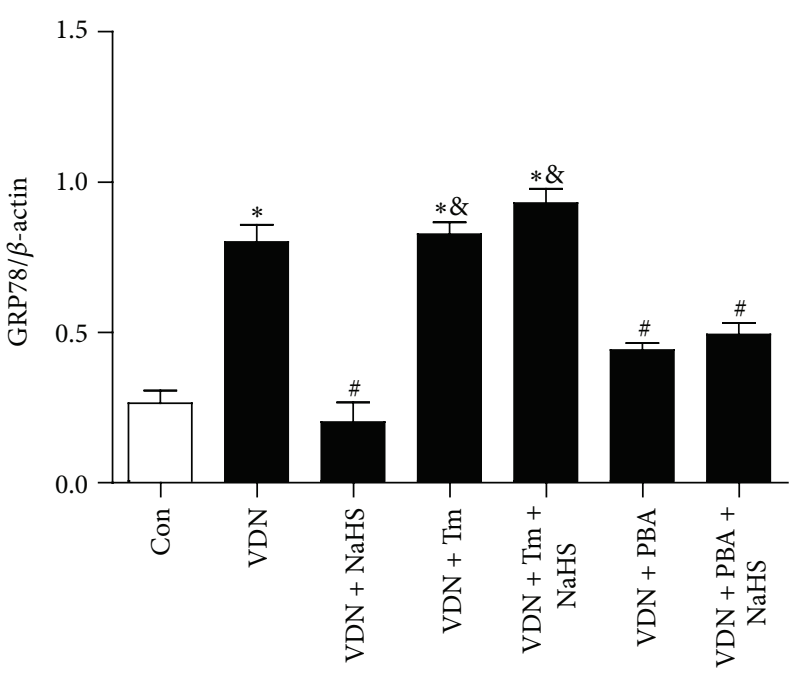

(b)

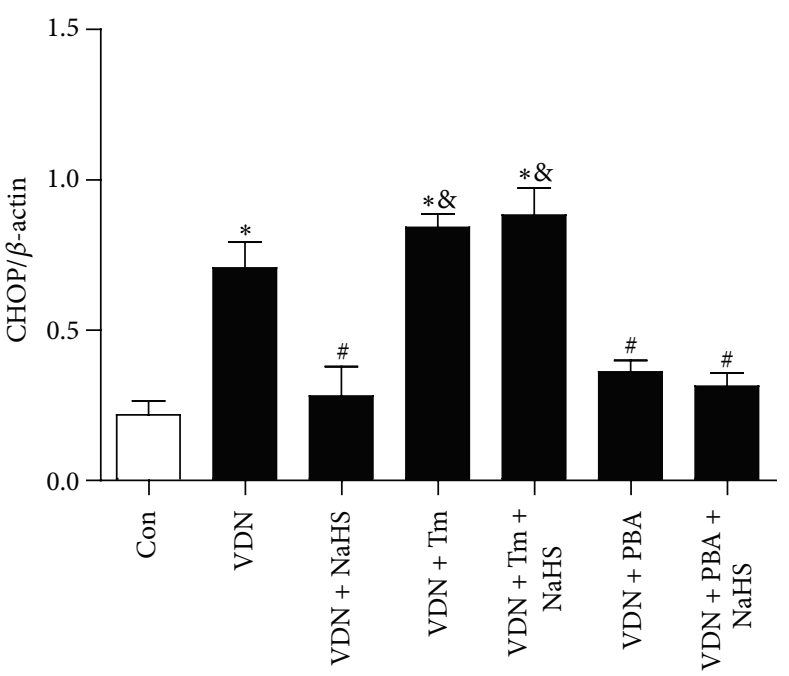

(d)

FIGURE 3: Tm blocks the effect of $\mathrm{H}_{2} \mathrm{~S}$ on activation of ERS in calcified aorta. (a) Representative protein levels of GRP78, active caspase-12, $\mathrm{CHOP}$, and $\beta$-actin; (b-d) quantitative analysis of protein levels of GRP78, active caspase-12, and CHOP. ${ }^{*} P<0.05$ cf. control group; ${ }^{*} P<0.05$ cf. VDN group; ${ }^{\&} \mathrm{P}<0.05 \mathrm{cf}$. VDN $+\mathrm{H}_{2} \mathrm{~S}$ group.

the VDN + NaHS group appeared to have less thickening and structural disorder of vascular elastic fibers (Figure 1), higher protein levels of SM22 $\alpha$ and calponin, and lower protein levels of BMP2 and RUNX2 (Figure 2).

Compared with the VDN rats, the VDN + NaHS rats had lower levels of the ERS markers GRP78, active caspase-12, and CHOP (Figure 3).

3.3. ERS Involved in the Ameliorated Effect of NaHS on VC in VDN Rats. The ERS inducer Tm and the ERS inhibitor PBA were used to investigate the role of ERS in VC. Tm could block the ameliorated effect of NaHS on VDN rats. Tm increased the ALP activity and calcium content (Table 1), downregulated the protein level of SM22 $\alpha$ and calponin, and upregulated the protein level of BMP2 and RUNX2 in aorta of VDN rats with NaHS treatment (Figure 2). The protein level of GRP78, active caspase-12, and CHOP was also induced by $\operatorname{Tm}$ (Figure 3). However, there was no difference between $\mathrm{VDN}$ rats and $\mathrm{VDN}+\mathrm{Tm}$ rats.

The effect of PBA on VC in VDN rats was similar as that of NaHS. PBA treatment also could decrease the ALP activity and calcium content in calcified aorta (Table 1). In VDN plus PBA rats, the downregulation of SM22 $\alpha$ and calponin was reversed, and the upregulation of BMP2 and RUNX2 was simultaneously decreased (Figure 2). The increased protein level of GPR78, active caspase-12, and CHOP in calcified aorta was inhibited by PBA treatment (Figure 3). There was no synergistic effect of NaHS plus PBA on VDN rats.

\subsection{Regulation of Akt Signaling Pathway in VC. Compared} with the control rats, the protein levels of Akt and phosphoAkt were both lower in the VDN rats. NaHS treatment significantly reversed the downregulation of Akt and phospho-Akt (Figure 4). 


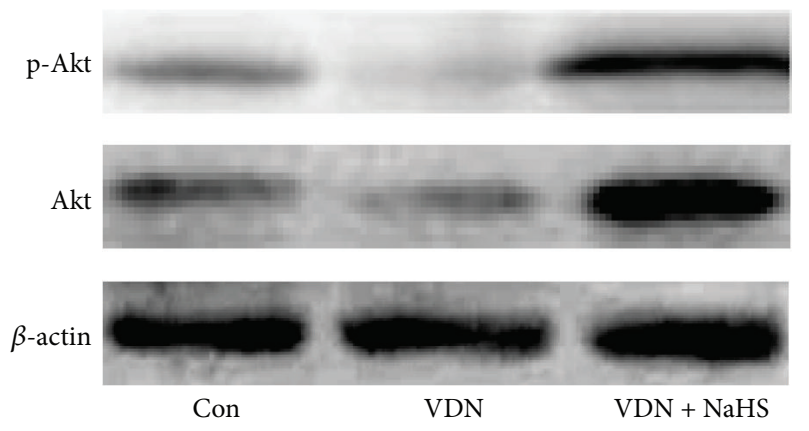

(a)

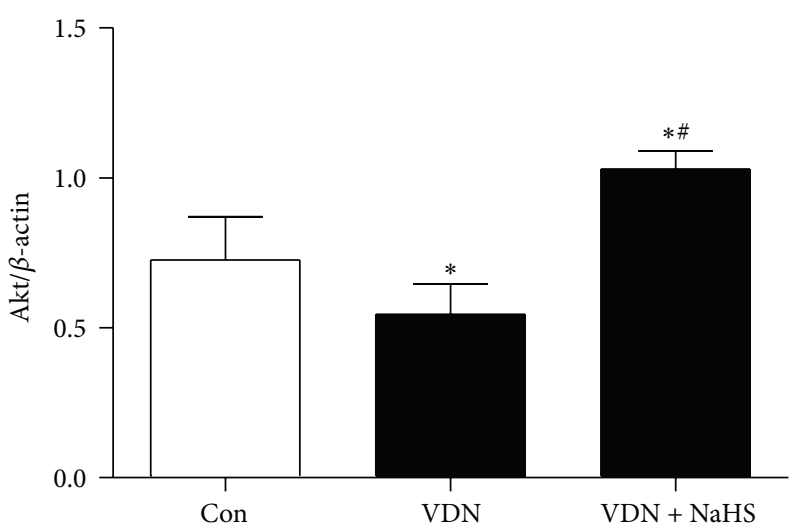

(c)

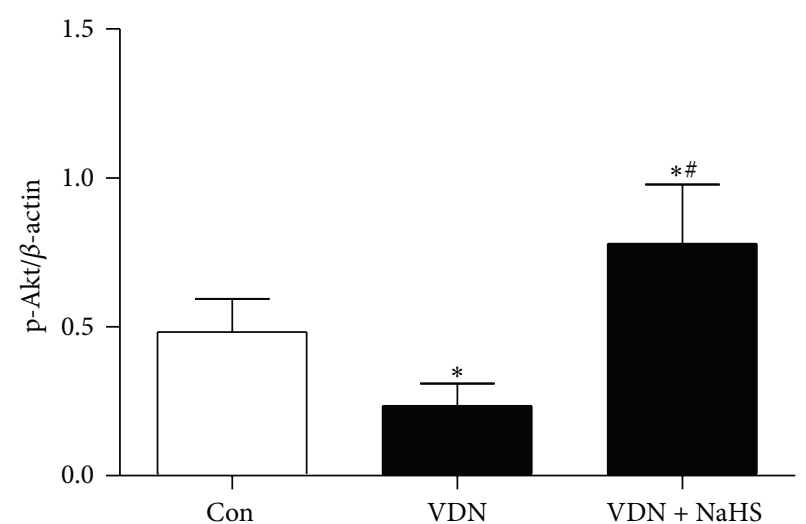

(b)

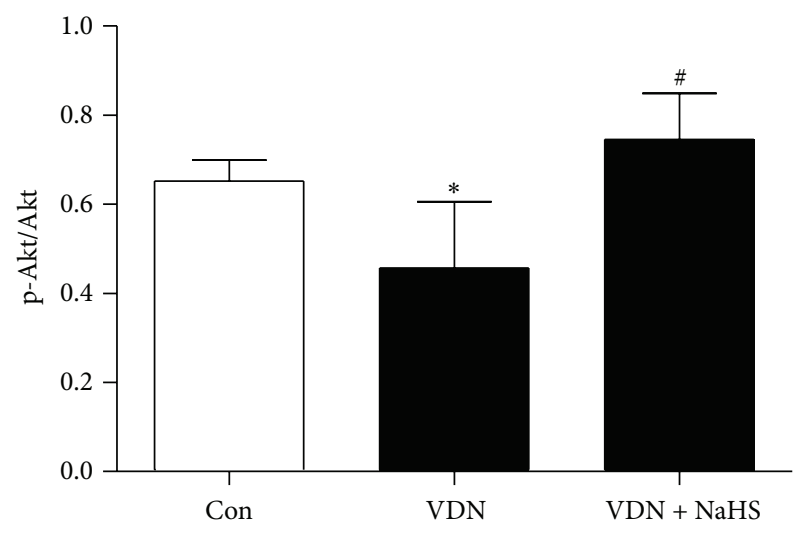

(d)

FIGURE 4: Regulation of Akt signaling pathway in aorta. (a) Representative protein levels of phospho-Akt, Akt, and $\beta$-actin; (b-d) quantitative analysis of protein levels of phospho-Akt and Akt. ${ }^{*} P<0.05 \mathrm{cf}$. control group; ${ }^{\#} P<0.05 \mathrm{cf}$. VDN group.

3.5. Protein Levels of Cystathionine $\gamma$-Lyase (CSE) in the Aorta and Plasma Levels of $\mathrm{H}_{2} \mathrm{~S}$. In the VDN rats, the protein levels of CSE in the aorta and plasma levels of $\mathrm{H}_{2} \mathrm{~S}$ were lower than that in the control rats. NaHS treatment could significantly increase the protein levels of CSE and plasma levels of $\mathrm{H}_{2} \mathrm{~S}$ in VDN rats (Figure 5).

\section{Discussion}

In this study, a rat model of VC was established using the VDN method, to investigate the effect of $\mathrm{H}_{2} \mathrm{~S}$ on VC and the phenotype transformation of VSMCs. In addition, $\mathrm{H}_{2} \mathrm{~S}$ can also inhibit ERS of calcified aortic tissues. The effect of $\mathrm{H}_{2} \mathrm{~S}$ on alleviating VC and the phenotype transformation of VSMCs can be blocked by Tm. PBA also alleviated VC and phenotype transformation of VSMCs, similar to the effect of $\mathrm{H}_{2} \mathrm{~S}$. Activation of Akt signaling may be involved in the ameliorated effect of $\mathrm{H}_{2} \mathrm{~S}$ on ERS and VC. Exogenous NaHS treatment upregulated the protein expressions of aortic CSE and plasma levels of $\mathrm{H}_{2} \mathrm{~S}$ in VDN rats. These results suggest that $\mathrm{H}_{2} \mathrm{~S}$ may alleviate VC of the rat aorta by inhibiting ERS. Thus, $\mathrm{H}_{2} \mathrm{~S}$ may be a new target for research of the prevention and treatment of VC (Figure 6).
It was initially assumed that $\mathrm{VC}$ is a passive process, resulting from disturbance of calcium/phosphorus metabolism and consequent deposition of these minerals. However, a major breakthrough in recent studies on the mechanism of VC revealed that VC is actually an active biological process similar to bone development, which is highly regulated, preventable, and can be reversed. The transformation of VSMCs from a contractile to osteoblast-like phenotype has been observed in both the vessels of patients with hypertension, diabetes, or chronic renal failure, and in the rat VC model established with a high-calcium, high-phosphorus, and high vitamin $\mathrm{D}_{3}$ and nicotine diet $[18,19]$. In the present study, the calcium content and ALP activity of the VDN group were significantly higher than that of the control group. Western blot analysis showed that levels of the proteins that indicate a contractile phenotype (SM22 $\alpha$ and calponin-1) were significantly lower in the VDN group relative to that of the control, while levels of the proteins associated with an osteoblast-like phenotype (RUNX2 and BMP2) were significantly higher. $\mathrm{H} \& \mathrm{E}$ staining revealed that, compared with the control group, the aortic elastic plate of the VDN group was significantly thicker and more disordered, with breakage of the elastic fibers. These results indicated that construction of the rat 


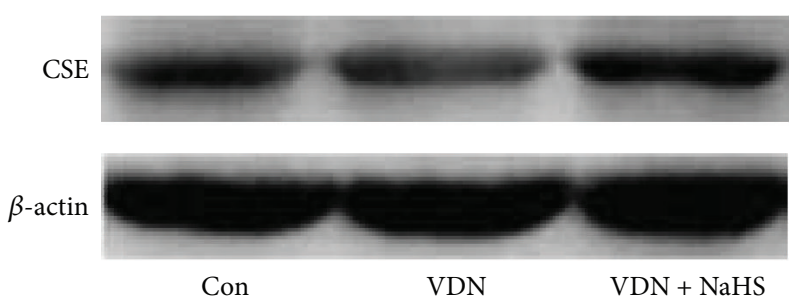

(a)

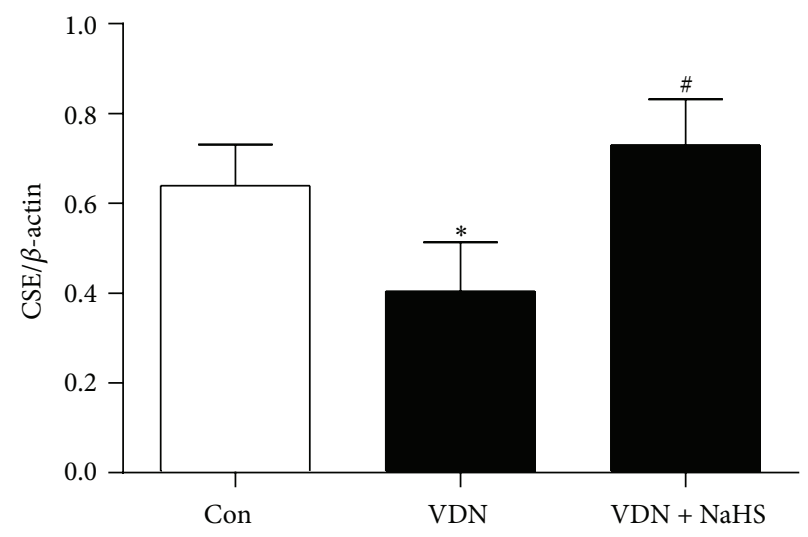

(b)

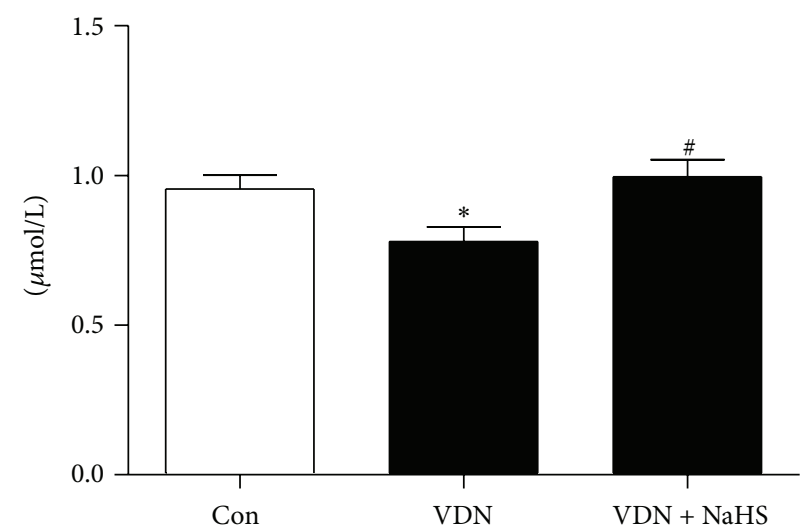

(c)

Figure 5: Protein levels of CSE in aorta and plasma levels of $\mathrm{H}_{2} \mathrm{~S}$. (a) Representative protein levels of CSE and $\beta$-actin; (b) quantitative analysis of protein levels of CSE. ${ }^{*} P<0.05$ cf. control group; ${ }^{\#} P<0.05 \mathrm{cf}$. VDN group.

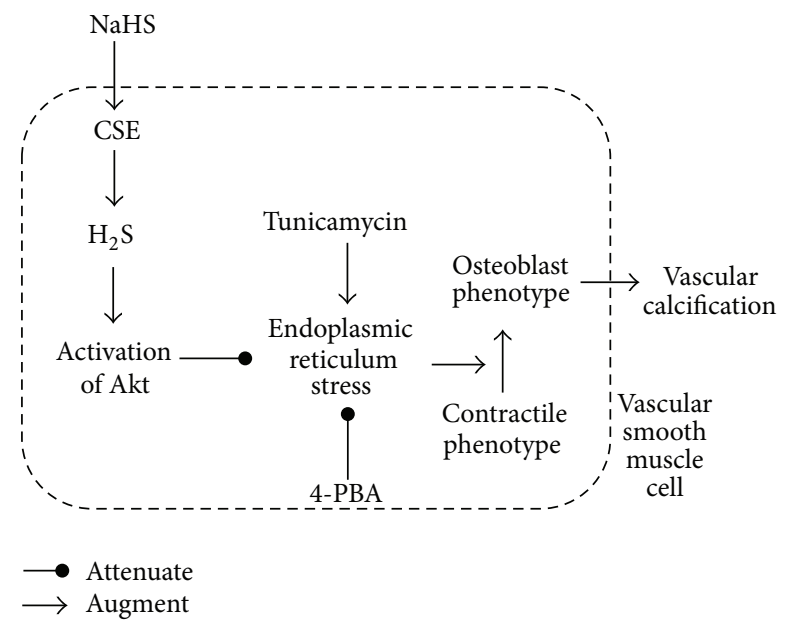

Figure 6: Schematic representation of the ameliorative effect of $\mathrm{NaHS}$ on VC.

VC model was successful and confirmed the transformation of VSMCs from a contractile to osteoblast-like phenotype during $\mathrm{VC}$.

$\mathrm{H}_{2} \mathrm{~S}$ is a newly identified gaseous signaling molecule after nitric oxide and carbon monoxide. $\mathrm{H}_{2} \mathrm{~S}$ has a rotten egg-like odor and is toxic, by inhibiting cytochrome C oxidase [20]. In vitro studies have shown that $\mathrm{H}_{2} \mathrm{~S}$ can reduce extracellular calcium deposition and inhibit expression of the genes responsible for the osteoblast-like transformation of VSMCs. In addition, $\mathrm{H}_{2} \mathrm{~S}$ also inhibits upregulation of the sodium-phosphate cotransporter Pit-1, induced by uptake of phosphate and phosphoric acid. CSE inhibitor blocks these effects [11]. These results suggest that $\mathrm{H}_{2} \mathrm{~S}$ strongly inhibits phosphate-induced VSMC calcification and osteoblast-like differentiation.

In vivo and in vitro experiments both indicate that $\mathrm{H}_{2} \mathrm{~S}$ inhibits the phenotype transformation of VSMCs and alleviates VC [9]. In the present experiment, the calcium content of the aorta and ALP activity of the VDN group were both higher compared with that of the control group, while NaHS treatment significantly alleviated such increase. H\&E staining revealed thickening and structural disorder of the vascular elastic fibers in the VDN group, and NaHS treatment significantly alleviated these changes. In addition, NaHS upregulated levels of protein related to the contractile phenotype of the aortic VSMCs, while inhibiting levels of proteins responsible for osteoblast-like phenotype. These results further show that $\mathrm{H}_{2} \mathrm{~S}$ inhibits phenotype transformation in VSMCs and alleviates VC. 
Any stimulus that interferes with endoplasmic reticulum function (such as hypoxia, oxidative stress, nutrition deficiency, and calcium homeostasis disturbance) may lead to accumulation of unfolded or misfolded protein in the endoplasmic reticulum, leading to ERS. Studies have indicated that apoptosis of VSMCs is an important aspect of VC, while ERS-induced apoptosis is obviously active in the calcified rat aorta [20-22]. Inhibition of ERS-induced VSMC apoptosis significantly alleviates development of VC [23-25]. Bone morphogenetic protein (BMP) signaling is a key aspect of VC that has an important role in osteoblast-like differentiation of VSMCs. It has been reported that BMP2 promotes VSMC calcification through induction of oxidative stress and ERS, and subsequently production of XBP1 (X-box binding protein 1), which binds to the Runx2 (runt-related transcription factor 2) promoter and promotes Runx 2 expression [26]. In the present study, we also found that GRP78, CHOP, and active caspase12 levels were significantly elevated in the calcified rat aorta, while the ERS inhibitor significantly alleviated VC. This indicated that ERS participates in the development of VC. NaHS inhibited ERS in the calcified rat aorta, and the ERS agonist blocked this effect of NaHS. These results suggest that $\mathrm{H}_{2} \mathrm{~S}$ may alleviate VC by inhibiting ERS.

$\mathrm{H}_{2} \mathrm{~S}$ putatively activates Akt signaling [27-30], and activation of the Akt signaling pathway has a crucial role in counteracting ERS [31-33]. Several factors could inhibit ERS by activating Akt signaling, resulting in a protective effect on the cardiovascular system [34]. Our present results showed that the protein levels of phospho-Akt and Akt were both upregulated by $\mathrm{NaHS}$ treatment. This suggests that $\mathrm{H}_{2} \mathrm{~S}$ ameliorated ERS through activation of the Akt signaling pathway.

$\mathrm{H}_{2} \mathrm{~S}$ is synthesized endogenously by the catalysis of 3 enzymes: CSE, cystathionine- $\beta$-synthase, and 3-mercaptopyruvate sulfurtransferase. CSE has a crucial role in maintaining cardiovascular homeostasis. Wu et al. [6] and our research team have both demonstrated that the expression and activity of CSE were reduced in the calcified aorta, which resulted in reduction of endogenous $\mathrm{H}_{2} \mathrm{~S}$. We have further shown that NaHS treatment upregulated the protein levels of CSE. The stimulation effect of exogenous $\mathrm{H}_{2} \mathrm{~S}$ treatment on the production of endogenous $\mathrm{H}_{2} \mathrm{~S}$ is in accord with other published articles $[35,36]$.

\section{Conclusion}

In this study, using a rat model of $\mathrm{VC}$, we showed that $\mathrm{H}_{2} \mathrm{~S}$ significantly alleviates VC, probably by inhibiting ERS. This study provides new strategies and a target for prevention and treatment of VC.

\section{Conflict of Interests}

The authors declare that there is no conflict of interests regarding the publication of this paper.

\section{Authors' Contribution}

Rui Yang and $\mathrm{Xu}$ Teng contributed equally to this paper.

\section{Acknowledgments}

The authors thank Sheng Jin for his help during revision and Bo Tan for his excellent technical assistance in $\mathrm{H}_{2} \mathrm{~S}$ measurements. This work was supported by the National Natural Science Foundation of China (nos. 31171098 and 81100229), the Specialized Research Fund for the Doctoral Program of Higher Education of China (no. 20121323110008), the Program for New Century Excellent Talents in University from the Education Ministry of China (Grant NCET-07-0252), the Hebei Province for Innovation Talents Support Plan (Grant LJRC017), and the Province Natural Science Foundation of Hebei (no. C2012206063).

\section{References}

[1] P. Lanzer, M. Boehm, V. Sorribas et al., "Medial vascular calcification revisited: review and perspectives," European Heart Journal, vol. 35, no. 23, pp. 1515-1525, 2014.

[2] K. I. Boström, N. M. Rajamannan, and D. A. Towler, "The regulation of valvular and vascular sclerosis by osteogenic morphogens," Circulation Research, vol. 109, no. 5, pp. 564-577, 2011.

[3] J.-S. Shao, J. Cai, and D. A. Towler, "Molecular mechanisms of vascular calcification. Lessons learned from the aorta," Arteriosclerosis, Thrombosis, and Vascular Biology, vol. 26, no. 7, pp. 1423-1430, 2006.

[4] M. Abedin, Y. Tintut, and L. L. Demer, "Vascular calcification: mechanisms and clinical ramifications," Arteriosclerosis, Thrombosis, and Vascular Biology, vol. 24, no. 7, pp. 1161-1170, 2004.

[5] C. M. Shanahan, M. H. Crouthamel, A. Kapustin, and C. M. Giachelli, "Arterial calcification in chronic kidney disease: key roles for calcium and phosphate," Circulation Research, vol. 109, no. 6, pp. 697-711, 2011.

[6] S. Y. Wu, B. H. Zhang, C. S. Pan et al., "Endothelin-1 is a potent regulator in vivo in vascular calcification and in vitro in calcification of vascular smooth muscle cells," Peptides, vol. 24, no. 8, pp. 1149-1156, 2003.

[7] L. L. Demer and Y. Tintut, "Inflammatory, metabolic, and genetic mechanisms of vascular calcification," Arteriosclerosis, Thrombosis, and Vascular Biology, vol. 34, no. 4, pp. 715-723, 2014.

[8] J. Bełtowski, "Hydrogen sulfide in pharmacology and medicine-an update," Pharmacological Reports, vol. 67, no. 3, pp. 647-658, 2015.

[9] N. Subhash, R. Sriram, and G. A. Kurian, "Sodium thiosulfate protects brain in rat model of adenine induced vascular calcification," Neurochemistry International, vol. 90, pp. 193-203, 2015.

[10] S.-Y. Wu, C.-S. Pan, B. Geng et al., "Hydrogen sulfide ameliorates vascular calcification induced by vitamin $\mathrm{D}_{3}$ plus nicotine in rats," Acta Pharmacologica Sinica, vol. 27, no. 3, pp. 299-306, 2006.

[11] E. Zavaczki, V. Jeney, A. Agarwal et al., "Hydrogen sulfide inhibits the calcification and osteoblastic differentiation of vascular smooth muscle cells," Kidney International, vol. 80, no. 7, pp. 731-739, 2011.

[12] T. Itou, N. Maldonado, I. Yamada et al., "Cystathionine $\gamma$-lyase accelerates osteoclast differentiation: identification of a novel regulator of osteoclastogenesis by proteomic analysis," Arteriosclerosis, Thrombosis, and Vascular Biology, vol. 34, no. 3, pp. 626-634, 2014. 
[13] L. A. Barr, Y. Shimizu, J. P. Lambert, C. K. Nicholson, and J. W. Calvert, "Hydrogen sulfide attenuates high fat diet-induced cardiac dysfunction via the suppression of endoplasmic reticulum stress," Nitric Oxide, vol. 46, pp. 145-156, 2015.

[14] Z.-F. Chen, B. Zhao, X.-Y. Tang et al., "Hydrogen sulfide regulates vascular endoplasmic reticulum stress in apolipoprotein E knockout mice," Chinese Medical Journal, vol. 124, no. 21, pp. 3460-3467, 2011.

[15] H. Wei, R. Zhang, H. Jin et al., "Hydrogen sulfide attenuates hyperhomocysteinemia-induced cardiomyocytic endoplasmic reticulum stress in rats," Antioxidants \& Redox Signaling, vol. 12, no. 9, pp. 1079-1091, 2010.

[16] N. Niederhoffer, Y. V. Bobryshev, I. Lartaud-Idjouadiene, P. Giummelly, and J. Atkinson, "Aortic calcification produced by vitamin D3 plus nicotine," Journal of Vascular Research, vol. 34, no. 5, pp. 386-398, 1997.

[17] X. Shen, C. B. Pattillo, S. Pardue, S. C. Bir, R. Wang, and C. G. Kevil, "Measurement of plasma hydrogen sulfide in vivo and in vitro," Free Radical Biology and Medicine, vol. 50, no. 9, pp. 10211031, 2011.

[18] K. A. Hruska, "Vascular smooth muscle cells in the pathogenesis of vascular calcification," Circulation Research, vol. 104, no. 6, pp. 710-711, 2009.

[19] M. Y. Speer, H.-Y. Yang, T. Brabb et al., "Smooth muscle cells give rise to osteochondrogenic precursors and chondrocytes in calcifying arteries," Circulation Research, vol. 104, no. 6, pp. 733741,2009

[20] T. H. Milby and R. C. Baselt, "Hydrogen sulfide poisoning: clarification of some controversial issues," American Journal of Industrial Medicine, vol. 35, no. 2, pp. 192-195, 1999.

[21] X. Duan, Y. Zhou, X. Teng, C. Tang, and Y. Qi, "Endoplasmic reticulum stress-mediated apoptosis is activated in vascular calcification," Biochemical and Biophysical Research Communications, vol. 387, no. 4, pp. 694-699, 2009.

[22] S. Miyazaki-Anzai, M. Masuda, K. M. Demos-Davies et al., "Endoplasmic reticulum stress effector CCAAT/enhancerbinding protein homologous protein (CHOP) regulates chronic kidney disease-induced vascular calcification," Journal of the American Heart Association, vol. 3, no. 3, Article ID e000949, 2014.

[23] M. Masuda, T. C. Ting, M. Levi, S. J. Saunders, S. MiyazakiAnzai, and M. Miyazaki, "Activating transcription factor 4 regulates stearate-induced vascular calcification," Journal of Lipid Research, vol. 53, no. 8, pp. 1543-1552, 2012.

[24] X.-H. Duan, J.-R. Chang, J. Zhang et al., "Activating transcription factor 4 is involved in endoplasmic reticulum stressmediated apoptosis contributing to vascular calcification," Apoptosis, vol. 18, no. 9, pp. 1132-1144, 2013.

[25] J.-R. Chang, X.-H. Duan, B.-H. Zhang et al., "Intermedin153 attenuates vascular smooth muscle cell calcification by inhibiting endoplasmic reticulum stress via cyclic adenosine monophosphate/protein kinase A pathway," Experimental Biology and Medicine, vol. 238, no. 10, pp. 1136-1146, 2013.

[26] M. Liberman, R. C. Johnson, D. E. Handy, J. Loscalzo, and J. A. Leopold, "Bone morphogenetic protein-2 activates NADPH oxidase to increase endoplasmic reticulum stress and human coronary artery smooth muscle cell calcification," Biochemical and Biophysical Research Communications, vol. 413, no. 3, pp. 436-441, 2011.

[27] Z. Guo, C. S. Li, C. M. Wang, Y. J. Xie, and A. L. Wang, "CSE/ $\mathrm{H}_{2} \mathrm{~S}$ system protects mesenchymal stem cells from hypoxia and serum deprivation-induced apoptosis via mitochondrial injury, endoplasmic reticulum stress and PI3K/Akt activation pathways," Molecular Medicine Reports, vol. 12, no. 2, pp. 2128-2134, 2015.

[28] W.-J. Cai, M.-J. Wang, P. K. Moore, H.-M. Jin, T. Yao, and Y.C. Zhu, "The novel proangiogenic effect of hydrogen sulfide is dependent on Akt phosphorylation," Cardiovascular Research, vol. 76, no. 1, pp. 29-40, 2007.

[29] B. L. Predmore, D. Julian, and A. J. Cardounel, "Hydrogen sulfide increases nitric oxide production from endothelial cells by an Akt-dependent mechanism," Frontiers in Physiology, vol. 2, article 104, 2011.

[30] S. J. Feng, H. Li, and S. X. Wang, "Lower hydrogen sulfide is associated with cardiovascular mortality, which involves cPKC $\beta$ II/Akt pathway in chronic hemodialysis patients," Blood Purification, vol. 40, no. 3, pp. 260-269, 2015.

[31] P. Hu, Z. Han, A. D. Couvillon, and J. H. Exton, "Critical role of endogenous Akt/IAPs and MEK1/ERK pathways in counteracting endoplasmic reticulum stress-induced cell death," The Journal of Biological Chemistry, vol. 279, no. 47, pp. 49420 49429, 2004.

[32] R. Y. Dai, S. K. Chen, D. M. Yan et al., "PI3K/Akt promotes GRP78 accumulation and inhibits endoplasmic reticulum stress-induced apoptosis in HEK293 cells," Folia Biologica, vol. 56, no. 2, pp. 37-46, 2010.

[33] Y. Zhang, Z. Xia, K. H. La Cour, and J. Ren, "Activation of Akt rescues endoplasmic reticulum stress-impaired murine cardiac contractile function via glycogen synthase kinase- $3 \beta$-mediated suppression of mitochondrial permeation pore opening," Antioxidants and Redox Signaling, vol. 15, no. 9, pp. 2407-2424, 2011.

[34] X. Teng, J. Song, G. Zhang et al., "Inhibition of endoplasmic reticulum stress by intermedin(1-53) protects against myocardial injury through a PI3 kinase-Akt signaling pathway," Journal of Molecular Medicine, vol. 89, pp. 1195-1205, 2011.

[35] N. Qipshidze, N. Metreveli, P. K. Mishra, D. Lominadze, and S. C. Tyagi, "Hydrogen sulfide mitigates cardiac remodeling during myocardial infarction via improvement of angiogenesis," International Journal of Biological Sciences, vol. 8, no. 4, pp. 430441, 2012.

[36] M. Wang, Z. Guo, and S. Wang, "The effect of certain conditions in the regulation of cystathionine $\gamma$-lyase by exogenous hydrogen sulfide in mammalian cells," Biochemical Genetics, vol. 51, no. 7-8, pp. 503-513, 2013. 


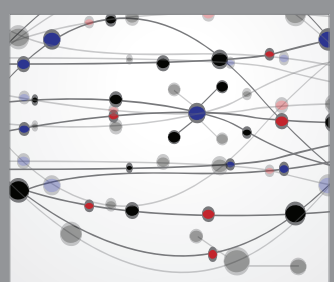

The Scientific World Journal
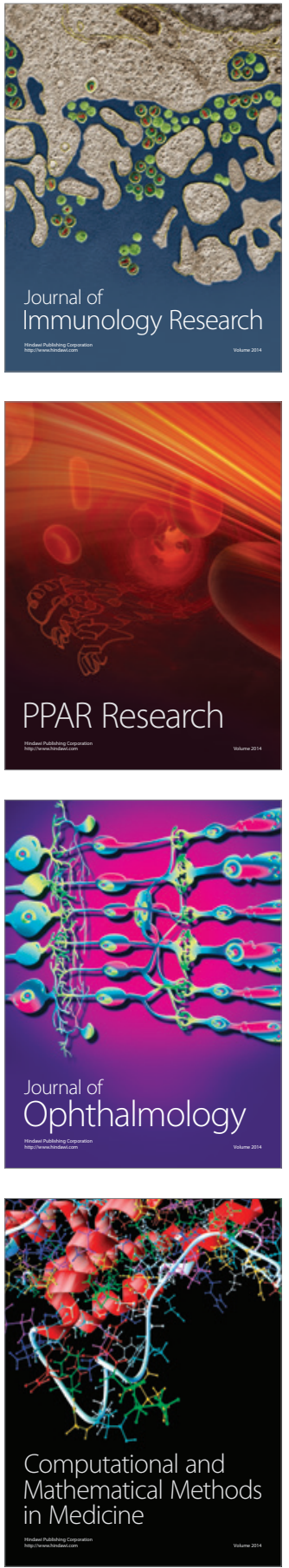

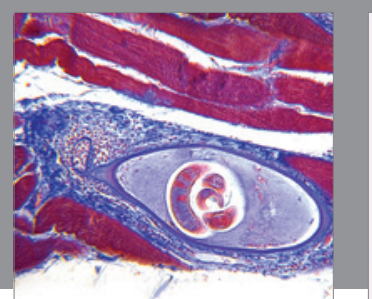

Gastroenterology Research and Practice

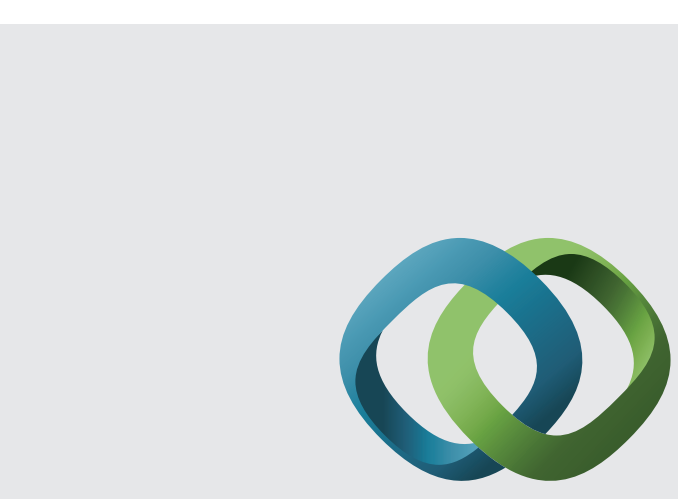

\section{Hindawi}

Submit your manuscripts at

http://www.hindawi.com
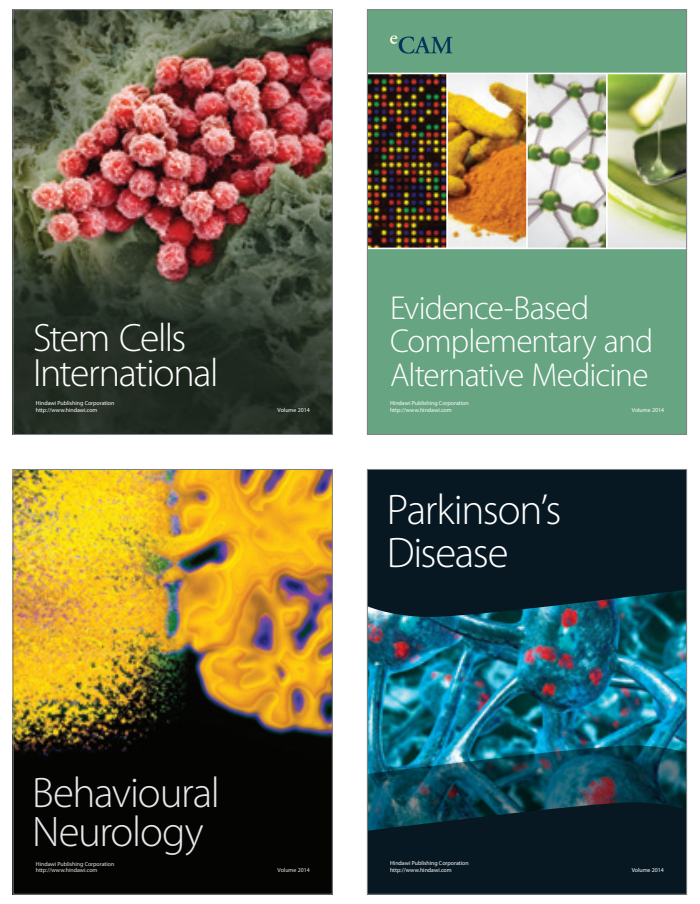
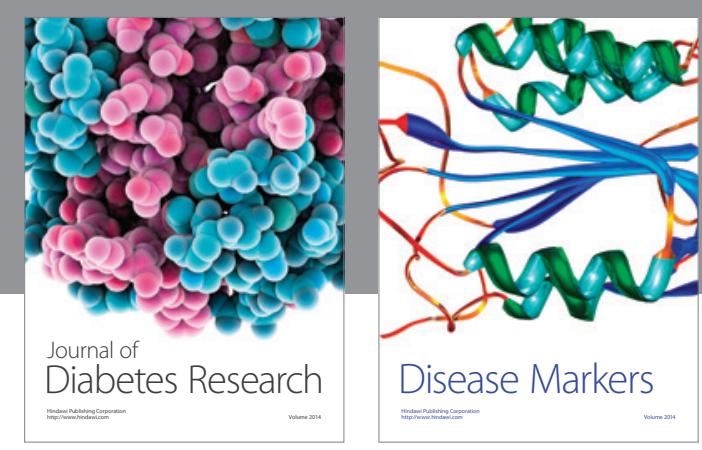

Disease Markers
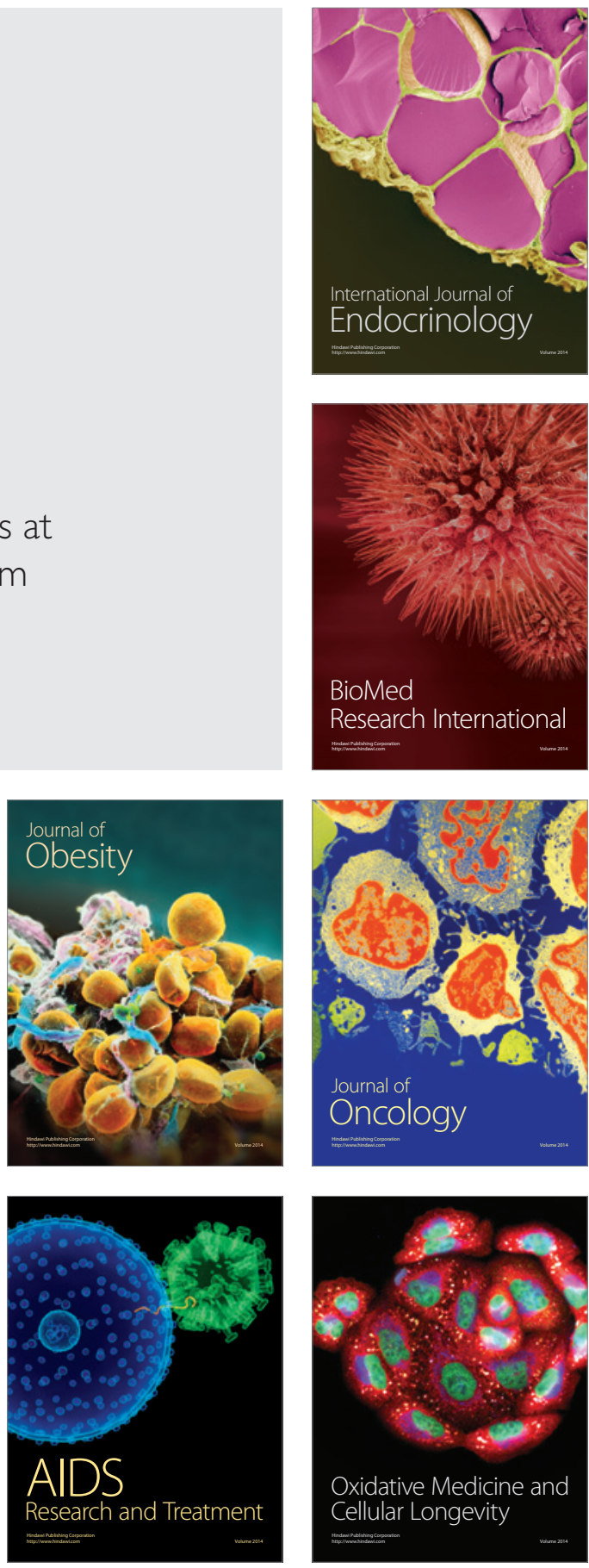